\title{
FUNDAMENTACIÓN EPISTEMOLÓGICA DE LOS DISEÑOS DE INVESTIGACIÓN NATURALISTA I. LA INVESTIGACIÓN-ACCIÓN. UN DISEÑO QUE SE ADAPTA AL ENTORNO ENFERMERO
}

\author{
ANA M. PIERA Orts \\ Lic. CC. de la Educación. \\ Hospital General Universitario de Valencia.
}

Correspondencia: Escalante, $18-3^{\circ}-6^{a} .46011$ Valencia

\section{RESUMEN}

Se presenta una fundamentación de los modelos que se instalan dentro del paradigma naturalista (también llamado fenomenológico, humanista, etnográfico...) de los que se señala el diseño de investigación-acción como propuesta para una indagación introspectiva colectiva en enfermería.

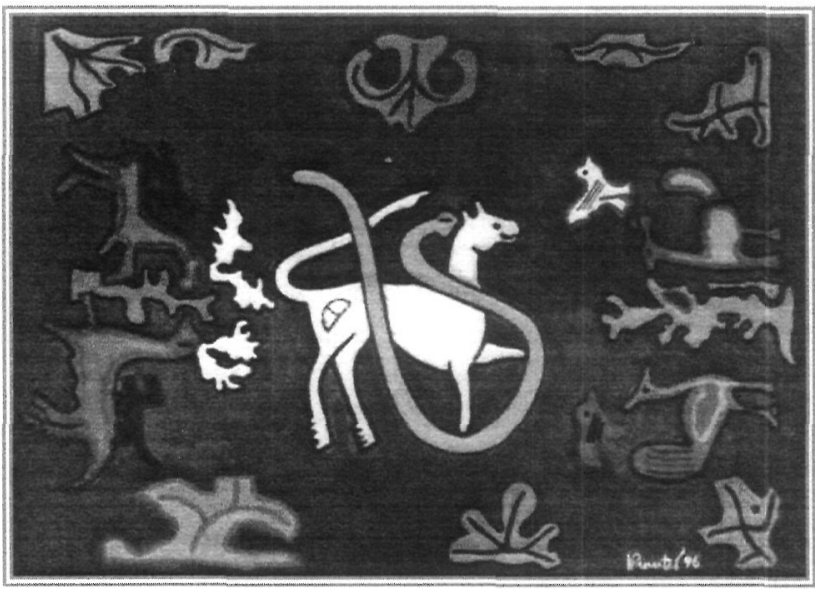

Javier de la Fuente

\section{FUNDAMENTS OF CERTAIN NURSING MODELS}

\section{SUMMARY}

Fundaments of certain nursing models embodied in a naturalistic paradigm (also called phenomenological, humanistic, ethnographical... prototypes) are presented here.

A proposal for specific action - a plan for introspective research in nursing - is also shown here.

\section{INTRODUCCIÓN}

A través de un breve recorrido por los ámbitos metodológicos de investigación se muestran las claves epistemológicas que fundamentan, desde diferentes ópticas, los requisitos de los procesos indagadores relativos a validez, fiabilidad y transferenciabilidad de los resultados de un estudio que, según sea el enfoque, tendrán una incidencia a diferente escala si se contemplan factores presentes en el nicho ecológico en el que se trabaja.

\section{ASPECTOS EPISTÉMICOS QUE} AFECTAN A LOS DISEÑOS DE INVESTIGACIÓN EN ENFERMERÍA

Durante un largo espacio de años, la polémica sobre paradigmas de investigación se ha centrado sobre las posibilidades de métodos cuantitativos versus cualitativos, como si con ello quedase suficientemente expresada la polémica de los diferentes paradigmas de investigación. Sin embargo, la diferencia no está, en si el método cualitativo describe 
mejor que el cuantitativo o viceversa; la diferencia radica en si el paradigma que asumimos, considera que nuestros hallazgos son o no neutrales, o lo que es lo mismo, si poseen valor universal o no.

En el sustrato de todo proceso intelectivo, queda siempre la opción del autor o responsable de orientarse paradigmáticamente en el modelo más acorde a su modo de interpretar el sustrato social y la concepción del hombre como ser social, individual y en proceso de cambio permanente. Cada opción o punto de vista personal, mantiene siempre una conexión explicativa o interpretativa de una concepción del mundo, que por personal que nos parezca, suele estar ya definida dentro de los ámbitos de la Filosofía, la Antropología, la Sociología o cualquier otra rama del saber, que se ocupe de describir los modelos de sociedad y el papel del hombre dentro de ellos.

Como espejo de esta situación, se aprecia que en la última década los modelos de gestión, asistencia técnico-profesional e investigador en enfermería se han acometido, generalmente, desde una óptica eficientista, que se ha servido de metodologías de carácter objetivista y especialmente servido por procesos explicativos de carácter numérico, que las más de las veces no han superado la localización de estadísticos básicos, a partir de los cuales o se operó poco o no se acompañó de explicación que fundamentase la causalidad pretendidamente hallada a través de la magia de los números.

En el fondo de la cuestión sobre la aceptación o no del un modelo de investigación, trabajo o proyección, se halla la fundamentación del posicionamiento científico que los sostiene. Pero veamos cual es la estructura del planteamiento científico más extendido. A la pregunta ¿qué es la ciencia? se viene respondiendo que "es una estructura asentada sobre hechos" (CHALMERS, 1987), y resumiendo la postura inductivista ingenua, se señala que la ciencia se basa en el principio de inducción, que podemos expresar así: si en una amplia variedad de condiciones se observa una gran cantidad de A y todos los A observados poseen sin excepción la propiedad $\mathrm{B}$, entonces todos los $\mathrm{A}$ tienen la propiedad $\mathrm{B}$; afirmando bajo estos presupuestos que la ciencia tiene capacidad de explicar y predecir, aplicando la lógica y razonamiento deductivo, cuando el cientí- fico tiene a su disposición leyes y teorías universales, pudiendo extraer de ellas, diversas consecuencias que le sirven como explicaciones y predicciones. Estos procesos deductivos se basan en la disciplina de la lógica bajo el esquema de dos proposiciones en las que dos premisas dan lugar de modo deductivo a una conclusión, también consideradas predicciones y explicaciones.

Este tipo de predicciones y explicaciones están conectadas a los modelos del tipo normativizador, en los que si P es deseable como finalidad y en las condiciones de un momento determinado, $\mathrm{Q}$ es el modelo más efectivo para conseguir Q, se recomienda entonces proceder según exige Q. Un modelo que si bien parece adaptarse a toda circunstancia social, en realidad queda inscrito en el grupo de aquellos donde o la cosa es susceptible de "sumarse y multiplicarse" o bien es susceptible de hallarse bajo unas condiciones, siempre controlables, como cuando se trata de las de un laboratorio, donde las variables son controladas. No se suele mencionar, en estos casos, que no todo lo social y humano es mensurable, ni tampoco se suele especificar la relevancia de aquellas variables que, por no mensurables, quedan fuera de control, o lo que es lo mismo, están descontroladas ejerciendo su influencia, sea cual sea su naturaleza.

Pero cuando un trabajo se da por concluido, se inicia una fase interrogatoria sobre la consistencia del mismo, y se plantean cuestiones sobre su validez, objetividad, transferibilidad, etc. Aspectos todos ellos que se enmarcan dentro de los llamados modelos científicos y cuya metodología establece los mecanismos para salvaguardar el rigor de los resultados a través de diferente tipo de técnicas. En este sentido, cabe plantear en primer lugar lo que entendemos por modelo científico, así como por aquellos términos cargados de cientificidad, que sin duda pueden formar parte de los elementos que posibiliten el arrumbamiento o no de cualquier trabajo.

Si bien es preciso insistir en el empleo del método científico como "mecanismo instrumental necesario para organizar de forma lógica los cuidados e impida el caos, la repetición y la falta de fundamento lógico de los mismos" (NOVALES et al., 1993, p. 43) y la consideración de que éste servirá 
como método a los cuidados de Enfermería en el denominado Proceso de Atención de Enfermería (PAE), hay que advertir que con relativa frecuencia se admite que el conocimiento profesional sólo será valioso cuando se trabaje con el método científico que, además de considerarlo como único es equiparado a un paradigma no explicitado.

Los esquemas utilizados habitualmente, aún sin que sea de forma explícita, vienen a coincidir con la estructura de un modelo de investigación, que consiste en el planteamiento de una hipótesis $\mathrm{H}$, a partir de la que tras la deducción se comprueba $\mathrm{D}$ y por tanto, queda provisionalmente establecida H (MORE, 1983), quedando definido este modelo explicativo y descriptivo como el modelo científico. Pero no siendo posible mantener este esquema para llevar a cabo todo tipo de investigaciones, en el campo de las disciplinas de carácter normativo, se suele estructurar el modelo, a partir del establecimiento de unos objetivos como finalidad, para lo que se buscará la forma más adecuada de conseguir el objetivo establecido, a partir de lo que serán estimadas las recomendaciones de carácter técnico, cuya efectividad previamente se han comprobado con un número determinado de sujetos, sobre los que han sido "comprobadas" unas relaciones causales, a partir del cálculo de probabilidad.

En ambos casos, la estructura está relacionada con la metodología que garantiza su carácter objetivo, lo que proporciona el valor a las conclusiones, en cuanto a su aplicabilidad, consistencia y neutralidad del propio investigador. Para ver la forma en que son transformadas las acciones instrumentales en acciones racionales, tomaremos el ejemplo de un sujeto al que bien se le interroga u observa, para descubrir como categoriza las opciones que toma.

Estas opciones naturalmente son acciones instrumentales que se pueden convertir en elecciones racionales si el sujeto, cuando las acomete, espera obtener el mejor resultado. Supongamos que interrogamos a un individuo sobre su preferencia entre manzanas y naranjas, y además le indicamos que, en su elección, puede contar tanto con la cualidad de las piezas como con la cantidad. El sujeto en cuestión nos responde que prefiere tres naranjas a cuatro manzanas, pero elige cinco manzanas sobre tres naranjas. Estas respuestas permiten elaborar una lista de comparaciones apareadas que recibe el nombre de ordenamiento de preferencias de los sujetos interrogados. Este listado de ordenamientos se complementa con otro al que se asignan los números más altos a aquellas opciones más preferidas por los sujetos, con lo que es posible elaborar matemáticamente una función de utilidad, a partir de la cual será factible el establecimiento de conclusiones sobre aquello que se indagó. Pero el ejemplo utilizado sobre preferencias de un cierto número de sujetos, aún siendo el resultado de acciones instrumentales, no tiene porque admitirse en todos los casos y, particularmente, en el ámbito de las ciencias sociales, no hay porque admitir sin más que sea necesariamente el resultado de acciones racionales, ya que la elección racional del sujeto, se ocupa de hallar el mejor medio para fines dados y ésto está vinculado a las creencias del propio sujeto y por tanto son falibles. (ELSTER, 1990).

Queda así expuesto, que el deficientemente denominado modelo científico, tanto se trate del explicativo como del normativo, recurre a la búsqueda de una objetividad, que no es la que nos va a informar de la realidad en el ámbito enfermero que nos ocupa.

Es precisamente el tratamiento personalizado de cada grupo de sujetos y la necesidad de que los proyectos que iniciemos mantengan la consistencia interna con su estructura teórica y metodológica, lo que nos obliga a reflexionar sobre "nuestros modos de hacer" investigador, gestor y fundamentalmente práctico-profesional. Prueba de la dispersión de modelos de actuación y su desconexión con los modelos de enfermería, que en las últimas décadas vienen avalados por la propia O.M.S., son esa multitud de trabajos de enfermería, donde el punto de arranque se presenta procedente de un modelo humanista y en los que, lamentablemente, es frecuente la aparición de metodologías de carácter racionalista y positivista mezcladas sin más criterio que el de acomodarse al mal denominado método científico de investigación, y presentando como métodos lo que no es más un grupo de herramientas, ascendiendo así a paradigma de investigación lo que no es más que un método entre tantos.

En definitiva pues, lo que parece claro, es que si en su conjunto las disciplinas sociales de una mis- 
ma área de conocimiento conviven entre si, simultaneándose o superviviendo diferentes paradigmas científicos (KUHN, 1987), el investigador debe conocer cuáles de las corrien-tes escoge, para informar sobre los diferentes aspectos a los que se refiere en su trabajo, debiendo integrarse todas, dentro de un mismo paradigma científico, que naturalmente comparten los colegios invisibles (POPKEWITZ, 1988).

Reflexionar sobre el hombre significa afrontar con los ojos abiertos el propio destino y plantearse claramente los problemas que resultan de la justa relación consigo mismo, con los demás hombres y con el mundo. Significa no solamente elaborar conceptos o idear sistemas, sino elegir, decidir, empeñarse, apasionarse: vivir auténticamente y ser auténticamente uno mismo.

Nadie puede pensar hoy que seamos capaces de conseguir realmente desde la ciencia positivista, la integración de un saber que posibilite las aplicaciones de conocimiento y mejora del sujeto desde una visión enfermera individualizada. Por este motivo, cabe una opción diferente, un método cualitativo -la investigación-acción- basado en Teorías Humanistas. Con esto no pretendemos enfrentarnos a otros métodos igualmente útiles, sino profundizar en una parcela de la ciencia, y estar abiertos a resultados y sugerencias, al adoptar una concepción del hombre como totalidad unitaria.

Plantear la reflexión sobre la práctica cotidiana de los actos enfermeros, como punto de partida para la mejora de la práctica profesional y de modo específico, a partir de diseños de investigación, nos sitúa dentro del paradigma naturalista. Este paradigma, que igualmente puede denominarse antropológico o etnográfico, es el que encaja, con los diseños llamados de INVESTIGACIÓN-ACCIÓN.

\section{CARACTERÍSTICAS}

\section{DE LA INVESTIGACIÓN-ACCIÓN}

Efectivamente los diseños de investigación-acción, como diseños emergentes que son, posibilitan la comprensión de los fenómenos que intervienen en la práctica cotidiana en entornos como el de enfermería, donde el problema no es determinar qué supuestos son verdaderos o no, sino cuáles se acomodan mejor al fenómeno bajo estudio. Así pues, el modelo de I-A diseñado por Kurt Lewin, supone la asunción de determinadas posturas, que merecen una pequeña revisión, al menos en lo que respecta a su adaptación a los entornos enfermeros, donde este modelo de indagación inicia sus primeras andaduras, al menos en lo que al territorio nacional se refiere.

El supuesto básico en el que descansa el proceso de la I-A, consiste en la posibilidad de diseñar planes flexibles y dúctiles, al objeto de someter la acción de los miembros del equipo pertenecientes a un mismo nicho ecológico, a una reflexión crítica. Sin embargo, para posibilitar una identificación y desarrollo de las interacciones entre la práctica, la investigación y la reflexión, en beneficio del conocimiento de la profesión, requiere una reflexión previa sobre la lógica interna de los presupuestos investigadores. La praxis profesional, la investigación y la reflexión conforman la tríada indispensable para el crecimiento de la profesión, lo que en términos epistemológicos no es otra cosa que la disposición hacia el desarrollo de conocimiento ideográfico, centrándose en las diferencias entre los objetos, tan frecuentemente y con tanto interés como en las similitudes, para comprender lo que sucede a nuestro alrededor y si las condiciones, son descritas de este modo, el paradigma naturalista es el que nos servirá como guía de orientación metodológica.

Esta ubicación paradigmática, lanza fuera de nuestras posibles metodologías, todas aquellas que mantengan la consideración de que el modelo científico es único, que todas cuantas hipótesis lancemos han de ser comprobadas y sólo admitidas las que sean generalizables, además de que nuestros instrumentos de medida serán los más exactos. Cualquiera de estas afirmaciones, propias del paradigma positivista, rompen con la lógica interna del paradigma naturalista, dentro del cual es acogido el diseño de investigación-acción.

Esta es la gran dificultad que el aprendiz de investigador, se encuentra en cuanto inicia su andadura: la de salvaguardar su trabajo de indagación de las dudas epistemológicas más habituales. Es propio que un gran número de enfermeras, formadas en la tradición positivista, considere que toda investi- 
gación que no se base en hipótesis contrastables y confirmables, sea desdeñable o exija mayor explicitación de los datos obtenidos, por el aparente escaso nivel de definición y/o conceptualización.

A partir de una situación problemática, en la que se perciben síntomas de desequilibrio, conflicto entre los profesionales o servicio y cuidados poco instaurados dentro de un modelo profesional dado, se podrá plantear una situación que, por dilemática, sea susceptible de estudio y no será suficiente decir que un trabajo contempla aspectos sociológicos, sino que además será necesario identificar, de entre los sociológicos, cuáles contempla y desde que óptica sociológica son examinados. En la misma medida cualquier definición relativa al ser humano, desde una visión psicológica, obviamente no será igualmente definida si el estudio se acomete desde una perspectiva skinneriana, piagetiana o rogeriana, etc.

Al plantear la elección sobre la adopción de un diseño investigador, que permita establecer el vínculo entre teoría y práctica y acercándonos más a modelos innovadores que posibiliten ejercer la función profesional en los órdenes tanto asistenciales, como docente-investigadores o gestores, nos encontramos con un hecho claro: enfermería es responsable de su actuación profesional y de mantener su competencia por medio de mejoras sucesivas. Las actividades de promoción y prevención han de cumplir una función social y para que esta se cumpla eficazmente, han de reconocer y admitir el valor social que el grupo da a la salud. Se ha de respetar la integridad del grupo, considerando y valorando las diferencias culturales, sociales y económicas.

El modelo de investigación-acción es difícil de encajar, puesto que asume los presupuestos de metodología de la etnografía, que arrancan desde las estructuras de Levis-Strauss y que es, en cierto modo el modelo participativo de Kemmis y Mactaggart. Este viene a vincularse en cierta medida al interaccionismo simbólico, posibilitando una especie de vínculo entre diferentes concepciones como la construcción social de Berger y Luckman, el análisis antropológico de Levis-Strauss, etc.
Los diseños de I-A tienen sus orígenes en la obra del psicólogo social Kurt Lewin (1946). Dos de sus principales ideas fueron las de decisión de grupo y compromiso con la mejora. La investigación-acción, por definición, es una actividad de grupo. Lewin la describe como una serie de fases en espiral, en las que se acometen sucesivamente la planificación, acción y evaluación del resultado (KEMMIS y MAcTAGGART, 1988), reiniciando de nuevo el proceso en una constante de retorno al inicio reflexivo que motivó la dirección de las decisiones del grupo.

Así también, posibilita que grupos de personas puedan organizar las condiciones en las que aprender de su propia experiencia. Este tipo de diseño permite planificar, actuar, observar y reflexionar de un modo cuidadoso, sistemático y sustantivamente riguroso, y siempre más adaptativamente que en la vida cotidiana, posibilitando además de los diferentes mecanismos del propio proceso reflexivo, como fuente de mejora y conocimiento de la realidad. Una realidad donde tienen lugar todos los procesos profesionales de carácter práctico, pero que se inspiran siempre en un conocimiento teórico y reflexivo que, con carácter recurrente, posibilita iluminar la práctica a través de la teoría e incrementar la teoría a partir de la observación del mundo de la praxis, donde de un modo empírico se opera y resuelve dificultades y nuevos casos de actuación profesional, donde las decisiones puestas en práctica, podrán ser recuperadas, reflexionadas y elaborado su fundamento y vínculo con el conjunto de aspectos teóricos que deberán quedar instaurados dentro un mismo modelo profesional.

Es obvio que, en el caso de las ciencias sociales, es factible el análisis de los intereses sociales y económicos a partir del estudio de los valores sociales y como ejemplo destacaremos la opinión de Popkewitz que plantea la importancia que tiene el lenguaje y afirma que la "teoría social puede tener la función de:

1. fundamentar el cambio de las condiciones sociales y económicas, haciendo que dicho cambio parezca razonable;

2. ofrecer un mecanismo de legitimación de intereses institucionales; 
3. guiar la creación de formas sociales alternativas" (POPKEWITZ, 1988, p. 40),

añadiendo, que estos intereses quedan en ocasiones en la sombra, a consecuencia de la forma discursiva de su "lenguaje formal y aparentemente universal", razón por la cual, la elección de la I-A posibilita desvelar las razones compartidas que realmente afectan a los grupos de trabajo, al proponer un cambio en alguno de sus ámbitos de actuación.

\section{DISEÑO DE PLANIFICACIÓN PARA LA ACCIÓN}

La primera cuestión que los investigadores deberemos revisar, es qué tipo de conocimiento precisamos generar y en combinación con nuestro modo de ver el mundo, esto es, nuestra concepción del hombre y de la sociedad, qué tipo de paradigma de investigación somos capaces de asumir. Este será el primer paso que podrá liberarnos de algunas de las incoherencias más frecuentes dentro de la lógica interna de los diseños de investigación.

El siguiente paso, consistiría en ubicar nuestro trabajo dentro de un marco teórico lo suficientemente concretado, como para que sirva de guía al posterior proceso que se inicia cuando acometemos una investigación.

Después de esta identificación, el grupo de investigadores establecerá el tópico sobre el que se estima necesario iniciar el proceso reflexivo. Inicialmente, será de esperar que el grupo de trabajo disponga de una baja conceptualización previa, a partir de la cual plantear las posibles guías de observación, análisis, reflexión y elaboración de constructos teóricos. Será paso previo pues, la identificación de los siguientes supuestos básicos:

\section{- Naturaleza de la realidad.}

- Relación investigadores-objeto.

- Naturaleza de los enunciados legales.

\section{- Métodos.}

- Criterios de calidad.

- Fuentes de la teoría.

- Instrumentos. todos ellos, al igual que las grandes teorías que definen al hombre y a la sociedad, se identifican con unas metodologías de investigación que a su vez se vinculan a los grandes paradigmas. Y será en este punto, en el que el grupo investigador habrá de ocuparse especialmente en mantener la coherencia del conjunto del trabajo, al identificar cada uno de estos supuestos, bajo la "mirada" que corresponde a la enfermera investigadora en la acción.

Como una guía para el proceso de investigación-acción, anotaremos aquí, aquellos aspectos que más sencilla-mente enlazan los diferentes supuestos básicos en que se apoyarán los diseños emergentes de este tipo de diseños investigadores, considerando que se insertan dentro de un paradigma naturalista y sin establecer la diferencia con el paradigma racionalista al que se enfrenta (GUBA, 1985), manteniendo el orden que arriba hemos expresado.

- Naturaleza de la realidad. Las investigadoras considerarán que a medida que la investigación avance y el conocimiento se incremente, le será implícito que las partes de la realidad se presenten interrelacionadas entre sí.

- Relación investigadores-objeto. Los investigadores en la acción considerarán que, entre ellos y los sujetos investigados, se darán cambios y la distancia entre si no será por tanto impenetrable.

- Naturaleza de los enunciados legales. El planteamiento naturalista acepta que las generaliza-ciones no son posibles, dado que se refieren a contextos particulares y por tanto desarrollan conocimiento ideográ-fico.

- Métodos. No hay que dotar de relevancia al método haciéndolo coincidir con métodos que puedan parecer más verosími-les, sino que cualquier método sea cuantitativo o cualita-tivo, servirá, si no rompen con el esquema de la naturaleza en que se describen los enunciados legales o la relación con el objeto.

- Criterios de calidad. Se considerará como criterio básico de calidad la relevancia del conocimiento derivado del proceso de investigación para el contexto donde se produjo. 
- Fuentes de la teoría. Se desestiman las hipótesis y se utilizan los modelos de contraste con la realidad.

- Instrumentos. Las investigadoras en la acción se utilizan a sí mismas, como instrumentos (observación, discusión, triangulación...), perdiendo objetividad pero ganando flexibili-dad.

A partir de la identificación de estos supuestos, el proceso de investigación-acción estará preparado para generar el suficiente conocimiento que procure la credibilidad, transferibilidad, dependencia del contexto y confirmabilidad que le son propios como modelo de investigación que se realiza en los nichos ecológicos a los que pertenece el grupo investigador y cuya finalidad será como indicábamos arriba la combinación vital para el avance de la profesión enfermera.

El desarrollo final dará lugar a un informe que contendrá, además de la compleja descripción del nicho ecológico donde se investigó, los resultados de las mejoras aplicadas como fruto del acuerdo al que llegó el grupo tras su proceso reflexivo compartido y siendo el informe de naturaleza ideográfica, será por tanto de mayor valor situacional para el origen donde tuvo lugar este proceso de compromiso compartido y se presentará como informe etnográfico. Su efecto de generabilidad se centrará, fundamentalmente, en las posibilidades de mejora a que dio lugar y su aplicación será exclusiva para su propio ámbito de origen, dando lugar a sucesivos procesos de investigación-acción.

\section{BIBLIOGRAFÍA}

Berger, P.; Luckmann, T. (1994) La construcción social de la realidad. Amorrortu Editores. $1^{a}$ edición en castellano 1968. Buenos Aires.

Chalmers, AF. (1987) ¿Qué es esa cosa llamada ciencia?. Trad. E. .Pérez Sedeño y P. López Máñez. Siglo XXI editores. Quinta edición en castellano. ed.or.1976, Madrid.

Elster, J. (1990) Tuercas y tornillos. Una introducción a los conceptos básicos de las ciencias sociales. Ed. orig. 1989. Ed. Gedisa. Barcelona,

Guba, EG. (1985): Criterios de credibilidad en la investigación naturalista. En: Gimeno
Sacristán, J. y Pérez Gómez, A. La enseñanza: su teoría y su práctica. Akal Editor. $2^{a}$ edi-ción, Madrid: 148-168.

Kemmis, S; McTaggart, B. (1988) Cómo planificar la investigación-acción. Trad. por Salcedo, sobre la Tercera ed. revisada de 1987. Laertes, Barcelona.

Kuhn, TS. (1987) La estructura de las revoluciones científicas. Trad. por A. Contin. ed. original 1962. Fondo de Cultura Económica, Madrid.

More, TW. (1983) Introducción a la teoría de la educa-ción. Trad. por M.A. Quintanilla. ed.original 1974. Alianza Editorial, Madrid.

Novales, MV. (et. al.) (1993) Cuidados de Enfermería. Análisis y aplicación práctica. Conselleria de Sanitat i Consum. Generalitat Valenciana, Valencia.

Popkewitz, TS. (1988) Paradigma e ideología en investiga-ción educativa. Trad. por A. Ballesteros. Ed.orig. 1984. Mondadori, Madrid.

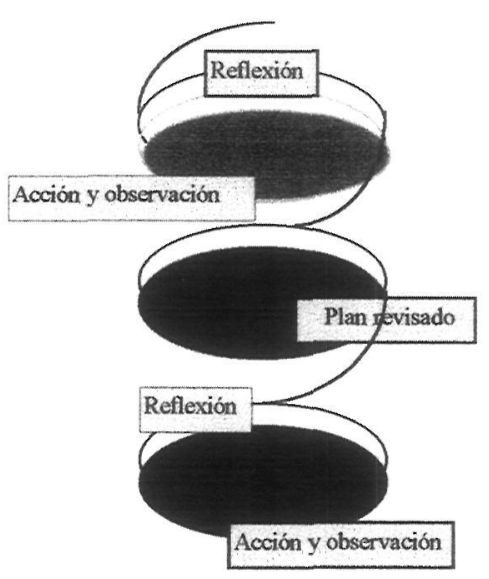

Tomado de Kemmis y Mactaggart, 1988, p.16

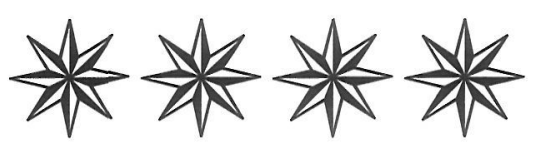

